\title{
Analytical study of the snap-through and bistability of beams with arbitrarily initial shape
}

\author{
Hussein Hussein \\ King Abdullah University of Science and Technology \\ Thuwal 23955-6900, Saudi Arabia \\ hussein.hussein@kaust.edu.sa \\ Mohammad I. Younis \\ Corresponding author \\ Physical Sciences and Engineering Division \\ King Abdullah University of Science and Technology \\ Thuwal 23955-6900, Saudi Arabia \\ mohammad.younis@kaust.edu.sa
}

\begin{abstract}
We derive the snap-through solution and the governing snapping force equations for an arbitrarily pre-shaped beam deflected under a mid-length lateral point force. The exact solution is obtained based on the classical theory of elastic beams as a superposition of the initial shape and the modes of buckling. Two kinds of solution are identified depending on the axial force level. The two solutions, bifurcation conditions, bistability conditions, and the snapping force equations are derived and discussed. The snap-through and snapping force solutions are then calculated for two common beam initial shapes, the curved (first buckling shape) and the inclined one (V-shape). In both cases, explicit expressions are obtained describing the snap-through behavior. The analytical modeling results show excellent agreement with the finite element simulations. The comparison between the two cases shows a similar snap-through behavior qualitatively, while several differences and similarities are noticed quantitatively.
\end{abstract}

\section{Introduction}

In the transition from macro-scale to micro-scale, the design of mechanisms and micro-structures is more constrained. The assembly is harder and common microfabrication techniques impose a monolithic constraint. Thus, mechanisms, which consist usually of an assembly of several rigid elements with joints at the macro-scale, are generally replaced by compliant mechanisms at the micro-scale.

Compliant mechanisms perform their function through the elastic deformation of their structures. Despite their limited elastic range of deformation, compliant mechanisms are attractive for many applications, even at the macro-scale. This is due to their several advantages, such as energy storage, reduced cost, improved accuracy and reliability, eliminated wear, friction and backlash, and the fact that they do not need assembly or lubrication.

Compliant bistable mechanisms have a range of deflection between two stable equilibrium configurations. These mechanisms have a double well potential behavior where the deformation energy is stored between two minima at the stable positions. Bistable beams with buckled-like shapes are the most common types of compliant bistable mechanisms.

Bistable beams exhibit additional advantages, such as their simplicity, passive holding, low actuation energy, small footprint, large stroke with small restoring forces, and negative stiffness zone. These advantages make bistable beams suitable for an increasing number of applications at different scales, such as space applications [1], biomedical [2], energy harvesting [3,4], resonators [5], actuators [6] accelerometers [7], shock sensors [8], gas sensors [9], pressure sensors [10], flow sensors [11], grippers [12], mechanisms with large displacement and small actuation stroke [13], switches [14], relays [15], memory devices [16], logics [17], lamina emergent frustrum [18], statically-balanced mechanisms [19], soft robotics [20], constant force mechanisms [21,22], bistable positioning [23-26], and multistable devices [27-32].

The shape of bistable beams can be realized by initial buckling or can be deliberately pre-shaped during fabrication. The buckling can be obtained by axial compression [33-35], heating expansion [36-38], or residual stresses [39-41]. A straight beam after buckling is always bistable and the snap-through behavior is symmetric between the two sides of buckling.

In contrast, due to the shifted initial state of pre-shaped beams, a second stable position exists only under some conditions on the initial shape. Further, whenever the pre-shaped beams have a second stable side, the stability margin is usually more important in their first stable side. Both force and displacement ranges are limited in the second stable side 
compared to the first one. Pre-shaped bistable beams with both ends fixed are widely used in microsystems due to their ease of fabrication without the need of external forces or hinged boundaries to induce buckling.

Pre-shaped beams can be used in single or parallel configurations depending on the application. A single beam configuration is usually used in electrostatic actuation [42, 43] where large dynamic deflection is required. The parallel configuration is more used in quasi-static applications where the bistability, straight guided displacement, negative stiffness, or other snap-through properties are desirable.

In the parallel configuration, several pre-shaped beams are connected by a shuttle in their mid-length, which constraints the displacement of the shuttle to a single direction. This constraints non-symmetrical modes of buckling, which enhances the bistability of these mechanisms [44,45].

Pre-shaped beams in parallel configurations are generally actuated with a lateral force applied on the shuttle. This is the case of interest for the present study. Other modes of actuation are more used for bistable beams with induced buckling, such as electromagnetic forces [46], moments applied in different locations [47], or lateral forces applied at different points along the beam length $[48,49]$.

Pre-shaped bistable beams can be classified as uniform or hinged beam structures. The uniform beam has a simpler structure with uniform cross-section. However, the hinged beam has a variable cross-section along the beam length. The hinged shape is usually designed to promote some snapthrough properties, such as symmetric bistability [50-53] or energy dissipation [54]. Uniform cross-section beams allow a more distributed compliance along their length. Hinged beams in contrast are constrained in their narrow parts where the deformation stress is more concentrated. This makes the use of uniform beams more efficient for miniaturization [45]. The study in this paper focuses on the case of uniform beams. Most reported uniform pre-shaped bistable beams have curved or inclined initial shapes. The curved shape is usually similar to the first mode shape of buckling of a straight beam. The inclined shape (V-shape) is easier to fabricate or assemble at macro-scale. Figure 1 shows the different pre-shaped beam types and configurations.

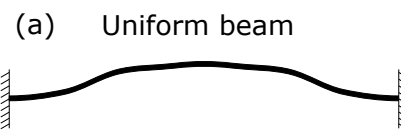

(c) Inclined beam
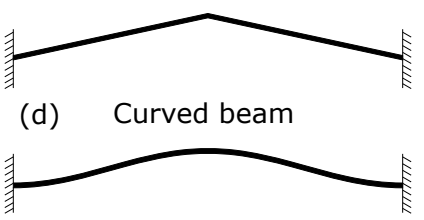

Fig. 1. Examples of pre-shaped bistable beams.

Many analytical models have been reported for each kind of bistable beams and for the different modes of ac- tuation. The modeling of bistable beams must account for the geometric nonlinearities associated with the simultaneous lateral deflection and axial compression and the scenarios of bifurcation in the snap-through behavior.

For pre-shaped beams with a mid-length lateral force, Qiu et al. [44] developed a modal superposition solution for the initially curved shape based on energy variation calculation and the classical beam theory. This work showed a bifurcation in the snap-through behavior of the curved beam where the second or third mode of buckling becomes activated at a certain level of the axial stress. Using the same derivation, Hussein et al. [55] developed explicit analytical expressions of the snapping forces and internal stresses that account for all modes of buckling. A generalized model for arbitrarily initial shape and loading is developed in [56] and solved using two different iterative algorithms. A bilateral relationship between the first and second stable configurations of bistable pre-shaped beams was formulated in [57].

For initially inclined beams, Zhao et al. [58] developed an elliptic integral model based on large deformation theory where shear and axial deformation are negligible. This model is effective for thin and flexible beams where the effects of axial elongation and shear are negligible [59]. In the same context, Holst et al. [60] considered the axial deflection in their elliptic integral model. A curve decomposition method is presented in [61] to simplify the large deformation analysis. Chen et al. [62] used a beam constraint model from [63] and added a shear effect correction based on the Timoshennko theory to predict the snapping forces. The constraint beam model was further extended in $[64,65]$. All cited modeling studies showed very good agreement with experiments and finite element method (FEM) simulations.

This work presents analytical study for the snap-through of an arbitrarily pre-shaped beam, subjected to a mid-length lateral point force. Explicit analytical expressions are derived describing the snap-through behavior, snapping forces and conditions for bifurcation, negative stiffness behavior, and bistability. The modeling is based on the classical beam theory, where the initial shape is expanded in Fourier series. The exact solution for the snap-through equation is derived considering all modes of buckling.

The snap-through governing expressions contain infinite sums depending on the modes of buckling and Fourier series coefficients. Some of these sums are already evaluated in [55], while the other sums are dependent on the initial shape. The infinite sums are evaluated for two initial shape cases, curved and inclined, and explicit analytical snap-through expressions are obtained. The analytical modeling results are compared with FEM simulations for selected cases.

The rest of the paper is organized as follows. The snapthrough solution for pre-shaped beams with arbitrarily initial shape is developed in Section 2. The snapping force solution for pre-shaped beams is then developed in Section 3. The calculations for a specific initial beam shape are clarified in Section 4 and the cases of pre-shaped curved and inclined beams are investigated. The snap-through behavior of both beam shapes is finally compared and discussed in Section 5. 
2 Snap-through solution of a pre-shaped beam

The snap-through solution for a pre-shaped beam with an arbitrarily initial shape is developed in this section.

\subsection{Problem definition and annotations}

Figure 2 shows a schematic of a clamped-clamped preshaped beam deflected under a mid-length lateral point load $f$. The beam has a span $l$, in-plane thickness $t$, initial middle height $h$, and out-of-plane depth $b$.

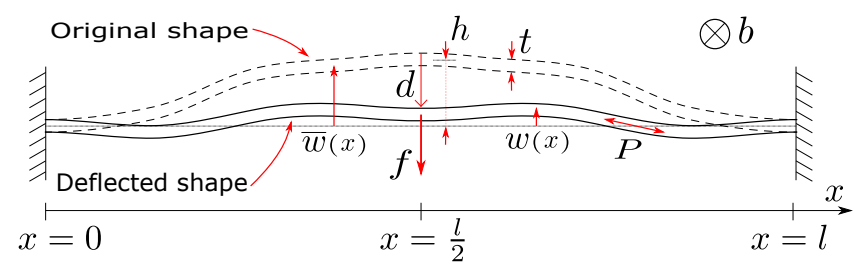

Fig. 2. Schematic of a clamped-clamped pre-shaped beam.

The shape of the beam is described by $w(x)$, which represents the shape of the beam along the $x$ axis. $\bar{w}(x)$ refers to the original as-fabricated beam shape at zero deflection without any residual stress. The following assumptions are considered for the beam shape in this study:

- Clamped-clamped boundary conditions ( $l$ is constant, $w(x)=\bar{w}(x), \frac{d w}{d x}(x)=\frac{d \bar{w}}{d x}(x)$ for $\left.x=0, l\right)$.

○ An initial middle height $h\left(\bar{w}\left(\frac{l}{2}\right)=h\right)$.

- Uniform beam shape ( $b$ and $t$ are constants).

- Small deformation hypothesis $(t, h \lll l)$.

- Symmetry of the initial shape with respect to the beam mid-length $\left(\bar{w}\left(\frac{l}{2}-x\right)=\bar{w}\left(\frac{l}{2}+x\right)\right)$. This is usually the case for parallel configurations.

The deflection $d$ at the beam mid-length is defined as:

$$
d=\bar{w}\left(\frac{l}{2}\right)-w\left(\frac{l}{2}\right)
$$

After deflection due to an applied point force $f$, the total length of the beam $s$ becomes contracted and the internal axial force $P$ appears in the beam. In elastic structures, the axial force is calculated using Hooke's law as follows:

$$
P=E A\left(\frac{\bar{s}-s}{\bar{s}}\right)
$$

where $\bar{s}$ is the initial beam length, $E$ is the Young's modulus and $A$ is the cross-section area.

Considering small deformation, the length $s$ of the beam can be approximated as follows:

$$
s=\int_{0}^{l} \sqrt{1+\left(\frac{d w}{d x}\right)^{2}} d x \approx l+\frac{1}{2} \int_{0}^{l}\left(\frac{d w}{d x}\right)^{2} d x
$$

For convenience, we normalize the various variables as follows:

$$
\begin{gathered}
X=\frac{x}{l} \quad W(X)=\frac{w(x)}{h} \quad S=\frac{s l}{h^{2}} \quad Q=\frac{h}{t} \\
N=\sqrt{\frac{P l^{2}}{E I}} \quad \Delta=\frac{d}{h} \quad F=\frac{f l^{3}}{E I h}
\end{gathered}
$$

where $I$ is the cross-section quadratic moment.

Considering uniform cross-section and material properties, the static snap-through behavior of a pre-shaped beam subjected to an axial force and a lateral point load is governed by:

$$
\frac{d^{4} W}{d X^{4}}-\frac{d^{4} \bar{W}}{d X^{4}}+N^{2} \frac{d^{2} W}{d X^{2}}=4 F \sum_{j=1,5,9 \ldots} \cos N_{j} X
$$

The mathematical derivation of (5) is presented in Appendix A. $N_{j}$ is the $j^{t h}$ critical buckling load. Mathematically, $N_{j}$ is calculated to have a non-trivial solution after introducing the boundary conditions in the homogeneous problem (similar to the problem of a straight beam). For clampedclamped boundary conditions, $N_{j}$ is the $j^{\text {th }}$ positive solution of the following periodic equation $[44,55]$ :

$$
\begin{aligned}
& \sin \frac{N_{j}}{2}\left(\tan \frac{N_{j}}{2}-\frac{N_{j}}{2}\right)=0 \\
& \begin{cases}N_{j}=(j+1) \pi & j=1,3,5 \ldots \\
N_{j}=2.86 \pi, 4.92 \pi, 6.94 \pi, 8.95 \pi \ldots j & =2,4,6 \ldots\end{cases}
\end{aligned}
$$

\subsection{Solution of the problem}

In order to solve (5), $W(X)$ is decomposed into three parts, the initial shape $\bar{W}(X)$, a particular solution $W_{p}$, and a homogeneous solution $W_{h}$.

$$
W(X)=\bar{W}(X)+W_{p}(X)+W_{h}(X)
$$

The consideration of the initial shape $\bar{W}(X)$ accounts for the inhomogeneous boundary conditions. Thereby, introducing (8) into (5), the problem can be decomposed into two separate problems with zero boundary conditions to find a particular and a homogeneous solution. The homogeneous problem is independent from the initial shape and external loads and is governed by the following equation:

$$
\frac{d^{4} W_{h}}{d X^{4}}+N^{2} \frac{d^{2} W_{h}}{d X^{2}}=0
$$

Equation (9) is the same equation governing a straight beam with an axial load. Its solution is an infinite superposition of the modes of buckling as follows $[44,55]$ :

$$
W_{h}(X)=\sum_{j=1}^{\infty} A_{j} W_{j}(X)
$$


where $W_{j}(X)$ is the $j^{t h}$ mode shape of buckling and $A_{j}$ is a constant that represents the contribution of the $j^{\text {th }}$ mode of buckling in the homogeneous solution. For clampedclamped boundary conditions, $W_{j}(X)$ is expressed as follows:

$$
\begin{cases}W_{j}(X)=1-\cos N_{j} X & j=1,3,5 \ldots \\ W_{j}(X)=1-\cos N_{j} X-2 X+\frac{2 \sin N_{j} X}{N_{j}} j=2,4,6 \ldots\end{cases}
$$

Further, introducing (8) into (5), the particular problem is governed by the following equation:

$$
\frac{d^{4} W_{p}}{d X^{4}}+N^{2}\left(\frac{d^{2} W_{p}}{d X^{2}}+\frac{d^{2} \bar{W}}{d X^{2}}\right)=4 F \sum_{j=1,5,9 \ldots} \cos N_{j} X
$$

The initial shape $\bar{W}(X)$ is usually symmetric between the two sides of the beam length. In parallel configurations, this symmetry serves for enhancing the bistability and to produce a straight deflection of the shuttle at the mid-length of the beam. An arbitrarily initial shape, which is symmetric with respect to the beam mid-length, can be expanded using Fourier series over the beam length as follows:

$$
\bar{W}(X)=C_{0}+\sum_{j=1,3,5 \ldots} C_{j} \cos \left(N_{j} X\right)
$$

where $C_{0}$ and $C_{j}$ are calculated as follows:

$$
\left\{\begin{array}{l}
C_{0}=\int_{0}^{l} \bar{W} d X \\
C_{j}=2 \int_{0}^{l} \bar{W} \cos \left(N_{j} X\right) d X
\end{array}\right.
$$

Introducing the second derivative of (13) into (12), the particular problem equation becomes

$$
\begin{aligned}
\frac{d^{4} W_{p}}{d X^{4}}+N^{2} \frac{d^{2} W_{p}}{d X^{2}} & =\sum_{j=1,5,9 \ldots}\left(4 F+C_{j} N_{j}^{2} N^{2}\right) \cos N_{j} X \\
& +\sum_{j=3,7,11 \ldots} C_{j} N_{j}^{2} N^{2} \cos N_{j} X
\end{aligned}
$$

For each term $\cos \left(N_{j} X\right)$ in (15), a particular solution $W_{p j}(X)$ satisfying zero boundary has the form $W_{p j}(X)=$ $B_{j} W_{j}(X)$. Thereby, the particular solution has the following form:

$$
W_{p}(X)=\sum_{j=1,3,5 \ldots} B_{j} W_{j}(X)
$$

where $B_{j}$ is a constant that represents the contribution of the $j^{t h}$ mode of buckling in the particular solution. $B_{j}$ are obtained by substituting (16) into (15):

$$
\begin{cases}B_{j}=\frac{4 F+C_{j} N_{j}^{2} N^{2}}{N_{j}^{2}\left(N^{2}-N_{j}^{2}\right)} j=1,5,9 \ldots \\ B_{j}=\frac{C_{j} N^{2}}{N^{2}-N_{j}^{2}} & j=3,7,11 \ldots \\ B_{j}=0 & j=2,4,6 \ldots\end{cases}
$$

Thereby, combining (8), (10), and (16), the exact solution of the equilibrium equation in (5) has the following form:

$$
W(X)=\bar{W}(X)+\sum_{j=1}^{\infty} K_{j} W_{j}(X)
$$

where $K_{j}=A_{j}+B_{j}$.

The terms $A_{j}$ can be calculated by introducing (10) into (9), which leads to the following:

$$
\sum_{j=1}^{\infty} N_{j}^{2}\left(N^{2}-N_{j}^{2}\right) A_{j} \cos \left(N_{j} X\right)=0
$$

According to (19), there is no contribution from the homogeneous solution when $N$ is not at any of the critical buckling loads $N_{j}$ :

$$
A_{j}=0 \text { for } N \neq N_{j}
$$

The same conclusion can be extracted by minimizing the variation of the total energy of the system as in $[44,55]$. Let $u_{b}, u_{c}, u_{f}$ and $u_{t}$ are the bending, compression, actuation and total energy and let $U_{(\cdot)}=\frac{u_{(\cdot)} l^{3}}{E I h^{2}}$ is the normalization for each of them. The variation of $U_{b}, U_{c}, U_{f}$ are calculated as follows:

$$
\begin{aligned}
& \partial U_{b}=\frac{1}{2} \partial \int_{0}^{1}\left(\frac{d^{2} W}{d X^{2}}-\frac{d^{2} \bar{W}}{d X^{2}}\right)^{2} d X \\
& \partial U_{c}=-N^{2} \partial S \\
& \partial U_{f}=-F \partial \Delta
\end{aligned}
$$

The variation of the total energy is the sum of bending, compression, and actuation energy variations:

$$
\begin{aligned}
\partial U_{t} & =\sum_{j=1,5,9 \ldots}\left(\left(N_{j}^{2}-N^{2}\right) K_{j}+\frac{4 F}{N_{j}^{2}}+C_{j} N^{2}\right) \frac{N_{j}^{2}}{2} \partial K_{j} \\
& +\sum_{j=3,7,11 \ldots}\left(\left(N_{j}^{2}-N^{2}\right) K_{j}+C_{j} N^{2}\right) \frac{N_{j}^{2}}{2} \partial K_{j} \\
& +\sum_{j=2,4,6 \ldots}\left(N_{j}^{2}-N^{2}\right) \frac{N_{j}^{2}}{4} \partial K_{j}^{2}
\end{aligned}
$$


Minimizing $\partial U_{t}$, the expressions of $K_{j}$ are obtained. In this case, it can be noticed that $K_{j}$ are equivalent to $B_{j}$ in (17) for $N \neq N_{j}$, which validates (20).

The energy variation calculation gives a further insight about the limitation of $N$. When $N$ starts to increase from zero after deflection between the two sides of buckling, $N$ cannot exceed $N_{2}$ physically since $\partial U_{t}$ is always positive. When $\mathrm{N}$ reaches $\mathrm{N}_{2}$ (if the geometry allows it), the second mode of buckling becomes activated and changes the snap-through behavior of the pre-shaped beam. Note that $N$ reaches higher limits for higher values of the height-tothickness ratio $Q$, as will be clarified latter.

The second mode can appear only in a single pre-shaped beam configuration, where a second stable position exists weakly only in some cases for high values of $Q$. In parallel configurations, the non-symmetrical modes of buckling are constrained, which enhances the bistability and allows a guided straight range of motion of the shuttle at the beams mid-length $[44,55]$. In this work, only the parallel configuration scenario is considered.

In parallel configurations, $N$ is able to exceed $N_{2}$ and a bifurcation between two kinds of solution emerges at the third critical buckling load $N_{3}$. In the first kind, the homogeneous solution has no contribution $\left(A_{j}=0\right.$ for $\left.j=1, \ldots, \infty\right)$ and it applies when $N$ is below $N_{3}\left(N<N_{3}\right)$. In the second kind, the third mode in the homogeneous solution becomes activated $\left(A_{3} \neq 0, A_{j}=0\right.$ for $\left.j \neq 3\right)$ and it applies when $N$ reaches $N_{3}\left(N=N_{3}\right)$. As can be noticed from (17), $N$ cannot reach $N_{3}$ if $C_{3} \neq 0$. Figure 3 demonstrates the variation of $N$ during deflection for two typical examples of pre-shaped curved beams. In the first example, $N$ cannot reach $N_{3}$ during deflection while $N_{3}$ is reachable in the second example. One can note from Fig. 3 that the beam, in both cases, is more compressed in the middle zone of deflection, as evident from the high values of $N$, while it is relaxed in the two sides of buckling. When $N_{3}$ is reached, the third mode of buckling appears in the beam shape preventing any further compression of the beam length. The occurrence of the two scenarios in Fig. 3 depends on the dimensional ratio $Q ; N$ can reach higher levels for higher values of $Q$. Hence, the third mode of buckling get involved during deflection for $Q$ higher than certain limit that will be determined in the next section.

In conclusion, the solution of the governing equation (5) has the form (18) where $K_{j}$ are expressed as follows:

$$
\begin{array}{ll}
\begin{array}{l}
\text { First kind } \\
N<N_{3}
\end{array} & \begin{cases}K_{j}=\frac{4 F+C_{j} N_{j}^{2} N^{2}}{N_{j}^{2}\left(N^{2}-N_{j}^{2}\right)} j=1,5,9 \ldots \\
K_{j}=\frac{C_{j} N^{2}}{N^{2}-N_{j}^{2}} & j=3,7,11 \ldots \\
K_{j}=0 & j=2,4,6 \ldots\end{cases} \\
\begin{array}{l}
\text { Second kind } \\
N=N_{3}
\end{array} & \begin{cases}K_{j}=\frac{4 F+C_{j} N_{j}^{2} N_{3}^{2}}{N_{j}^{2}\left(N_{3}^{2}-N_{j}^{2}\right)} j=1,5,9 \ldots \\
K_{j}=\frac{C_{j} N_{3}^{2}}{N_{3}^{2}-N_{j}^{2}} & j=7,11,15 \ldots \\
K_{j} \neq 0, C_{j}=0 & j=3 \\
K_{j}=0 & j=2,4,6 \ldots\end{cases}
\end{array}
$$

\subsection{First kind of solution}

Governing equations Introducing the solution from (18) and (23) into (1) and (2), the following equations are obtained governing the relationship between $F, N$ and $\Delta$ :

$$
F=\frac{1}{\Lambda_{e}(N)}\left(\Lambda_{d}(N)-\Delta\right)
$$

$$
\Lambda_{a}(N) F^{2}+\Lambda_{b}(N) F+\Lambda_{c}(N)+\frac{N^{2}}{12 Q^{2}}=0
$$

where $\Lambda_{a}, \Lambda_{b}, \Lambda_{c}, \Lambda_{d}$ and $\Lambda_{e}$ are infinite sum functions that are dependent on $N$ as follows: 


$$
\begin{aligned}
& \Lambda_{a}(N)=\sum_{j=1,5,9 \ldots .} \frac{4}{N_{j}^{2}\left(N^{2}-N_{j}^{2}\right)^{2}} \\
& \Lambda_{b}(N)=\sum_{j=1,5,9 . .} \frac{2 C_{j} N_{j}^{2}}{\left(N^{2}-N_{j}^{2}\right)^{2}} \\
& \Lambda_{c}(N)=\sum_{j=1,3,5 \ldots} \frac{-C_{j}^{2} N_{j}^{2} N^{2}}{4} \frac{N^{2}-2 N_{j}^{2}}{\left(N^{2}-N_{j}^{2}\right)^{2}} \\
& \Lambda_{d}(N)=\sum_{j=1,5,9 . .} \frac{-2 C_{j} N^{2}}{N^{2}-N_{j}^{2}} \\
& \Lambda_{e}(N)=\sum_{j=1,5,9 \ldots} \frac{8}{N_{j}^{2}\left(N^{2}-N_{j}^{2}\right)}
\end{aligned}
$$

The infinite sums $\Lambda_{a}$ and $\Lambda_{e}$ are independent from the initial shape. These sums were calculated in [55] for the case of a pre-shaped curved beam:

$$
\begin{aligned}
& \Lambda_{a}(N)=\frac{3}{16 N^{4}}\left(1-\frac{\tan \frac{N}{4}}{\frac{N}{4}}+\frac{\tan ^{2} \frac{N}{4}}{3}\right) \\
& \Lambda_{e}(N)=\frac{1}{N^{3}}\left(\frac{N}{4}-\tan \frac{N}{4}\right)
\end{aligned}
$$

Equation (25) is a second order polynomial equation of $F$ where the polynomial constants are dependent on $N$. Similarly, substituting (24) into (25), the resulting equation is a second order polynomial equation of $\Delta$ where the polynomial constants are dependent on $N$. This implies that for each value of $N$ in the first kind of solution, there are two corresponding values of $F$ and $\Delta$; as clarified for the latter in Figure 3.

Maximum limit of axial compression The axial force $N$ reaches a maximum level $N_{c}$ in the middle zone of deflection as can be seen from Figure 3. The value of $N_{c}$ increases with increasing the dimensional ratio $Q$. The specific ratio $Q_{c}$, which allows $N$ to reach a maximum limit $N_{c}$ during deflection, is the minimum of $Q$ that allows obtaining real solutions for $F$ at $N=N_{c}$ in (25). $Q_{c}$ is then expressed as follows:

$$
Q_{c}=N_{c} \sqrt{\frac{\Lambda_{a}\left(N_{c}\right)}{3 \Lambda_{b}^{2}\left(N_{c}\right)-12 \Lambda_{a}\left(N_{c}\right) \Lambda_{c}\left(N_{c}\right)}}
$$

Calling $F_{c}$ and $\Delta_{c}$ the corresponding values for $F$ and $\Delta$ for $N=N_{c}$ respectively; they can be determined from (24) and (25) as follows:

$$
\begin{aligned}
F_{c} & =-\frac{\Lambda_{b}\left(N_{c}\right)}{2 \Lambda_{a}\left(N_{c}\right)} \\
\Delta_{c} & =\Lambda_{d}\left(N_{c}\right)+\frac{\Lambda_{e}\left(N_{c}\right) \Lambda_{b}\left(N_{c}\right)}{2 \Lambda_{a}\left(N_{c}\right)}
\end{aligned}
$$

Note that the determination of $Q_{c}$ from (28) is limited to the range $N_{c} \in\left[0, N_{3}\right]$. Equation (28) helps to determine the geometrical conditions to reach the different levels of axial compression, to have negative stiffness snap-through behavior, and to have a second kind of solution zone. In the sequel, $Q_{1}, Q_{2}$ and $Q_{3}$ refer to the values of $Q_{c}$ for $N_{c}=N_{1}, N_{2}$ and $N_{3}$, respectively.

Lateral stiffness The lateral stiffness $S$ of the beam in the snap-through zone is defined as $(\partial f / \partial d)$, which is proportional to $(\partial F / \partial \Delta)$. The stiffness expression can be deduced from (24) as follows:

$$
S=\frac{E I}{l^{3}} \frac{\partial F}{\partial \Delta}=\frac{E I}{l^{3}} \frac{1}{\Lambda_{e}}\left(\frac{\partial N}{\partial \Delta}\left(\frac{\partial \Lambda_{d}}{\partial N}-F \frac{\partial \Lambda_{e}}{\partial N}\right)-1\right)
$$

The lateral stiffness $S$ (which is proportional to the natural frequency) decreases as the beam is compressed. This can be seen from the natural frequency as reported in [66]. Thus, in order to determine at which level the beam starts to show a negative stiffness behavior zone, we choose to calculate $S$ at the maximum axial stress point of deflection $\left(N=N_{c}\right.$, $\left.\Delta=\Delta_{c}, F=F_{c}\right)$. At this point, $(\partial N / \partial \Delta)$ is equal to zero by definition, which gives the following expression of the stiffness:

$$
S_{N=N_{c}}=\frac{E I}{l^{3}} \frac{-1}{\Lambda_{e}\left(N_{c}\right)}
$$

From the expression of $\Lambda_{e}$ in (27b), we can deduce that the stiffness $S_{N=N_{c}}$ is positive for $N_{c} \in\left[0, N_{1}\right.$ [, equal to zero for $N_{c}=N_{1}$ and is negative for $\left.\left.N_{c} \in\right] N_{1}, N_{3}\right]$. This means that a pre-shaped beam starts to show a negative stiffness behavior for a $Q$ value around $Q_{1}$. Figure 4 shows the typical snapping force curves for a pre-shaped curved beam in the three cases: $Q<Q_{1}, Q=Q_{1}$ and $Q_{1}<Q<Q_{3}$.

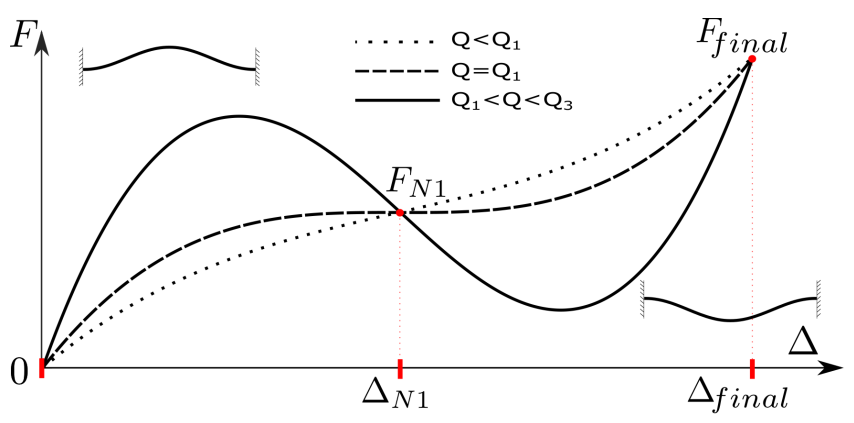

Fig. 4. Typical snapping force curves for a shallow pre-shaped curved beam with $Q<Q_{1}, Q=Q_{1}$ and $Q_{1}<Q<Q_{3}$. $Q_{1}$ and $Q_{3}$ refer to the values of $Q_{c}$ for $N_{c}=N_{1}$ and $N_{3}$, respectively.

As can be seen in Figure 4, $Q_{1}$ is the boundary between different behaviors in terms of the lateral stiffness. For lower values of $Q$, only positive stiffness behavior can be noticed. 
For $Q$ values close to $Q_{1}$, the beam acts as a constant force mechanism in the middle zone of deflection. However, this zero stiffness property is limited in terms of the range of displacement due to the small value of $Q_{1}$ in practice. For higher values of $Q$, the pre-shaped beam starts to show a negative stiffness behavior around the middle zone of deflection. This negative stiffness zone is a necessary but insufficient condition to have bistability. The condition for bistability is that the snapping force reaches negative values during deflection. In that case, the beam pushes itself to the second stable side instead of pushing back to the initial position. Note that the negative stiffness zone cannot be measured using a forcecontrolled experiment; a deflection control is required.

Calling $F_{N_{1}}$ and $\Delta_{N_{1}}$ the values of $F$ and $\Delta$, respectively, for $Q=Q_{1}$ and $N=N_{1}$; we can notice from Figure 4 that the snapping force curves for the different values of $Q$ for shallow beams $\left(Q<Q_{3}\right)$ pass in close vicinity of that point. $F_{N_{1}}$ and $\Delta_{N_{1}}$ can be calculated from (29) by setting $N_{c}=N_{1}$. Note that $F_{N_{1}}$ is only dependent on $C_{1}$ as follows:

$$
F_{N_{1}}=-4 \pi^{4} C_{1}
$$

Note also that the value of $F$ for $N=N_{1}$ remains equal to $F_{N_{1}}$, even for $Q>Q_{1}$.

End of compression The limit of the snap-through deflection zone where the solution developed in this paper remains applicable is the limit at which $N$ reaches zero after deflection. After this limit, the beam becomes in tension $(N<0)$, not in compression. Calling $\Delta_{\text {final }}$ and $F_{\text {final }}$ the values of $\Delta$ and $F$ at this limit, respectively; they can be deduced from (24) and (25) as follows:

$$
\begin{gathered}
\Delta_{\text {final }}=-160 \sum_{j=1,5,9 . .}\left(\frac{C_{j}}{N_{j}^{2}}\right) \\
F_{\text {final }}=-30720 \sum_{j=1,5,9 . .}\left(\frac{C_{j}}{N_{j}^{2}}\right)
\end{gathered}
$$

Note that $F_{\text {final }}$ is positive since it is proportional to $\Delta_{\text {final }}$, which is in the $\Delta$ positive zone $\left(F_{\text {final }}=192 \Delta_{\text {final }}\right)$.

\subsection{Second kind of solution}

For the second kind of solution, $N$ remains constant at $N_{3}$ for a certain range of deflection. We call $F_{3}$ the snapping force in the second kind of solution. The relation between $F_{3}$ and $\Delta$ in this range can be obtained from (24):

$$
F_{3}=64 \pi^{2}\left(\Lambda_{d 3}-\Delta\right)
$$

where $\Lambda_{b 3}, \Lambda_{c 3}$ and $\Lambda_{d 3}$ are used instead of $\Lambda_{b}\left(N_{3}\right), \Lambda_{c}\left(N_{3}\right)$ and $\Lambda_{d}\left(N_{3}\right)$ in the sequel, respectively.

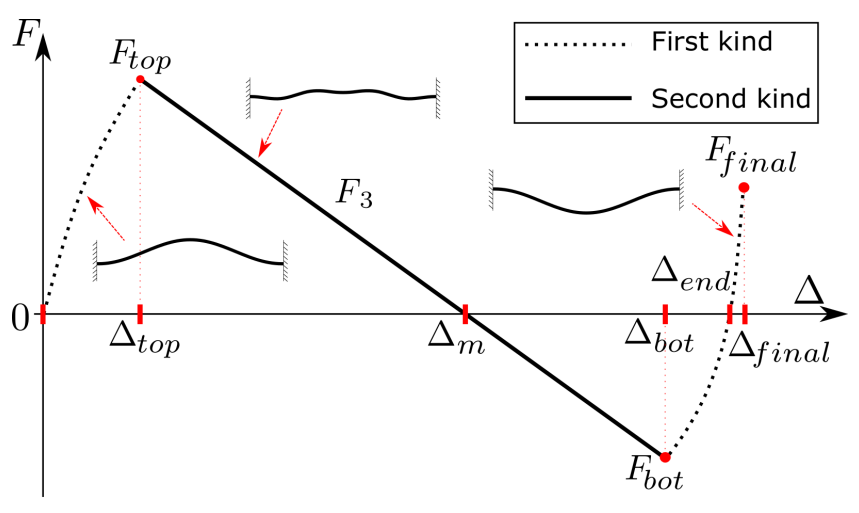

Fig. 5. Typical snapping force curve for a pre-shaped curved beam with $Q>Q_{3}$. Initially, the snapping force increases with increasing the beam mid-point deflection, maintaining a shape defined by the $1^{\text {st }}, 5^{\text {th }}, 9^{\text {th }} \ldots$ modes of buckling ( $1^{\text {st }}$ mode is dominating), until reaching $\Delta_{\text {top }}$. In between $\Delta_{\text {top }}$ and $\Delta_{b o t}$, the third mode of buckling involves in addition in the shape of the beam, resulting in a constant negative stiffness behavior. After $\Delta_{b o t}$, the third mode of buckling is no more involved and the snapping force increases with the deflection. The negative stiffness in-between $\Delta_{t o p}$ and $\Delta_{b o t}$ leads to bistability (two zero-force positions with positive stiffness behavior at $\Delta=0$ and at $\left.\Delta_{\text {end }}\right)$.

The second kind of solution zone is reachable during deflection for $Q \geq Q_{3}$. The typical snapping force curve for a pre-shaped curved beam in this case is shown in Figure 5.

From (34) and Figure 5, one can notice that whenever the initial shape of a pre-shaped beam allows reaching $N=N_{3}$, the lateral stiffness of the beam in the range of deflection of the second kind of solution $\left(S_{3}\right)$ is negative, constant and is only dependent on the material strength and the beam dimensions (except for the initial height $h$ ). However, $S_{3}$ is independent from the specific initial shape of the beam, as given below:

$$
S_{3}=-64 \pi^{2} \frac{E I}{l^{3}}
$$

The third mode constant $K_{3}$ in the second kind of solution can be calculated from the Hooke's law. Mathematically, $K_{3}$ is added in (25) in a way that $N$ remains equal to $N_{3}$. Recall that $C_{3}=0$ is a condition for $N$ to reach $N_{3}$. Thereby, $K_{3}$ is equivalent to:

$$
K_{3}= \pm \frac{1}{2 \pi} \sqrt{-\frac{3}{4096 \pi^{4}} F_{3}^{2}-\Lambda_{b 3} F_{3}-\Lambda_{c 3}-\frac{4 \pi^{2}}{3 Q^{2}}}
$$

Considering (34), $K_{3}$ can be expressed as a function of $\Delta:$

$$
K_{3}= \pm \frac{1}{2 \pi} \sqrt{\begin{array}{c}
-3 \Delta^{2}+\left(6 \Lambda_{d 3}+64 \pi^{2} \Lambda_{b 3}\right) \Delta-\frac{4 \pi^{2}}{3 Q^{2}} \\
-3 \Lambda_{d 3}^{2}-64 \pi^{2} \Lambda_{b 3} \Lambda_{d 3}-\Lambda_{c 3}
\end{array}}
$$


The limits of the second kind of solution zone in terms of $\Delta$ and $F$ can be obtained from the expression of $K_{3}$. We label $\left(\Delta_{\text {top }}, F_{\text {top }}\right)$ at the first limit (closer to $\left.\Delta=0\right)$ and $\left(\Delta_{b o t}, F_{b o t}\right)$ at the second limit. $\Delta_{t o p}, F_{t o p}, \Delta_{b o t}$ and $F_{b o t}$ are calculated at the limits where $K_{3}$ in (36) and (37) has a real solution $\left(K_{3}=0\right)$.

$\Delta_{\text {top }}=\Lambda_{d 3}+\frac{32 \pi^{2} \Lambda_{b 3}}{3}-\sqrt{\left(\frac{32 \pi^{2} \Lambda_{b 3}}{3}\right)^{2}-\frac{\Lambda_{c 3}}{3}-\frac{4 \pi^{2}}{9 Q^{2}}}$

(38a)

$F_{\text {top }}=64 \pi^{2}\left(\frac{-32 \pi^{2} \Lambda_{b 3}}{3}+\sqrt{\left(\frac{32 \pi^{2} \Lambda_{b 3}}{3}\right)^{2}-\frac{\Lambda_{c 3}}{3}-\frac{4 \pi^{2}}{9 Q^{2}}}\right)$

$\Delta_{b o t}=\Lambda_{d 3}+\frac{32 \pi^{2} \Lambda_{b 3}}{3}+\sqrt{\left(\frac{32 \pi^{2} \Lambda_{b 3}}{3}\right)^{2}-\frac{\Lambda_{c 3}}{3}-\frac{4 \pi^{2}}{9 Q^{2}}}$

$(38 \mathrm{c})$

$F_{b o t}=64 \pi^{2}\left(\frac{-32 \pi^{2} \Lambda_{b 3}}{3}-\sqrt{\left(\frac{32 \pi^{2} \Lambda_{b 3}}{3}\right)^{2}-\frac{\Lambda_{c 3}}{3}-\frac{4 \pi^{2}}{9 Q^{2}}}\right)$

The snapping forces at $F_{\text {top }}$ and $F_{b o t}$ are practically the maximum positive and minimum negative reachable force levels between the stable sides in case of bistability, respectively.

\subsection{Bistability of pre-shaped beams}

A bistable pre-shaped beam have a negative snapping force in some range of the snap-through deflection. The negative snapping force indicates that the beam is pushing itself to the other side of buckling. The bistability here refers to the ability of the beam to retain the static position without the application of external forces. In this case, the stable position has a zero snapping force value $(F=0)$.

An essential property for a stable position is the positive stiffness, which means that the beam pushes back to the stable position after a slight deflection. In these conditions, the two stable positions are $\Delta=0$ and $\Delta_{e n d}$, which satisfy zero snapping force and positive stiffness as shown in Figure 5. The second zero-force position $\Delta_{m}$ is an unstable position since the beam has a negative lateral stiffness around it. The values of $\Delta_{m}$ and $\Delta_{\text {end }}$ are calculated from (24) as follows:

$$
\begin{aligned}
\Delta_{m} & =\Lambda_{d}\left(N_{m}\right) \\
\Delta_{\text {end }} & =\Lambda_{d}\left(N_{\text {end }}\right)
\end{aligned}
$$

The existence of the unstable position $\Delta_{m}$ indicates the existence of a negative force zone and thereby the bistability (Figure 5). $\Delta_{m}$ can be located in both kinds of solution zone. However, the stable positions are always in the applicable zone of the first kind of solution since the stiffness in the second kind of solution is negative (35). The second stable position $\Delta_{\text {end }}$ has a positive value of $N$ (Figure $5, N_{\text {final }}=$ $0<N_{\text {end }}<N_{\text {bot }}=N_{3}$ ). Hence, it is sufficient to prove the existence of at least one zero force solution $F=0$ of the first kind of solution governing expression (25) in the range $N \in\left(0, N_{3}\right)$ to prove the bistability of a pre-shaped beam:

$$
\text { Bistability } \Longleftrightarrow \exists N \in\left(0, N_{3}\right), \Lambda_{c}(N)+\frac{N^{2}}{12 Q^{2}}=0
$$

Note that the existence of the second kind of solution is not necessary for bistability. Note also that in a single beam configuration (second kind of solution applies for $N=N_{2}$ ), the bistability condition is that a solution of (40) exists for $N \in\left(0, N_{2}\right)$.

The minimum of $Q$ allowing a pre-shaped beam to have bistability is the minimum of $Q$ that satisfies (40). Independently from $Q$, we can extract from (40) that $\Lambda_{c}(N)$ for a bistable pre-shaped beam must have a negative value for some portion of $N \in\left(0, N_{3}\right)$ in case of bistability.

The expression of $\Lambda_{c}(N)$ (26c) is an infinite sum of positive components for $N \in\left(0, \sqrt{2} N_{1}\right]$, while only the component multiplied by $C_{1}^{2}$ has a negative value in the range $N \in\left(\sqrt{2} N_{1}, N_{3}\right)$. Thereby, the condition of bistability in (40) can be reformulated as follows:

$$
\text { Bistability } \Longleftrightarrow \exists N \in\left(\sqrt{2} N_{1}, N_{3}\right), \Lambda_{c}(N)<0
$$

In conclusion, the first mode of buckling in the initial shape of a pre-shaped beam (proportional to $C_{1}$ ) must be sufficiently dominant over the other modes of buckling to have bistability (since only $C_{1}$ makes a negative contribution to satisfy (41)). This justifies the consideration of the curved shape as a common shape for a pre-shaped bistable beam. Further, in case (41) is satisfied, the minimum value of $Q$ for reaching bistability is the real minimum of $\sqrt{-N^{2} / 12 \Lambda_{c}(N)}$ in the range $N \in\left(\sqrt{2} N_{1}, N_{3}\right)$.

Furthermore, as the $j^{\text {th }}$ modes of buckling $(j=$ $3,7,11, \ldots)$ are only involved in $\Lambda_{c}(N)$ and add only positive contribution to $\Lambda_{c}(N)$, which degrades the bistability (41), an initial shape without these modes of buckling will have a better bistability. In other words, if a pre-shaped beam is bistable and the Fourier series expansion for the initial shape includes components $C_{j}$ for $(j=3,7,11, \ldots)$, neglecting these components while keeping the same components $C_{j}$ for $(j=1,5,9, \ldots)$ will result in a better bistability behavior. Considering the boundary conditions, only $C_{0}$ will be changed after neglecting $C_{j}$ for $(j=3,7,11, \ldots) . C_{0}$ in this case is equivalent to $1 / 2$. The simplest shape that satisfies this property is the inclined shape, which justifies its consideration as another common shape for a pre-shaped beam bistable mechanism. 


\section{Calculations for specific beam shapes}

In the previous sections, derivations are developed for a general case with an arbitrarily initial shape. Calculations for specific initial shapes are presented in this section.

For a specific initial beam shape $\bar{W}$, the first step is to determine the corresponding Fourier series coefficients $C_{j}(14)$. Coefficients $\Lambda_{b}, \Lambda_{c}$ and $\Lambda_{d}$ (26), are calculated for a specific initial shape. Then, the buckling mode constants $K_{j}((23)$ and (37)) are obtained. Introducing the different coefficients and constants, the snap-through solution (18) and snapping force expressions ((24), (25), (34)) are obtained. Afterwards, the different snapping points ((29), (32), (33), (38), (39)) and the conditions for reaching different levels of axial compression (28) and for bistability (41) are determined. These steps are summarized in Table 1.

Table 1. Computational steps for the initial shape of beams.

\begin{tabular}{|c|c|c|}
\hline & Steps of calculation & Equations \\
\hline 1 & Fourier coefficients $C_{0}$ and $C_{j}$ & (14) \\
\hline 2 & Coefficients $\Lambda_{b}, \Lambda_{c}$ and $\Lambda_{d}$ & (14) in (26) \\
\hline 3 & Buckling mode constants $K_{j}$ & $\begin{array}{l}\text { (14) in (23), } \\
\text { (26) in (37) }\end{array}$ \\
\hline 4 & Snap-through solution & (23), (37) in (18) \\
\hline 5 & Snapping force expressions & $\begin{array}{c}(26),(27) \text { in } \\
(24),(25),(34)\end{array}$ \\
\hline 6 & Snapping points & $\begin{array}{c}(14),(26),(27) \text { in } \\
(29),(32),(33), \\
(38),(39)\end{array}$ \\
\hline 7 & $\begin{array}{l}\text { Conditions for reaching } \\
\text { levels of compression }\end{array}$ & (26), (27) in (28) \\
\hline 8 & Bistability condition & (26) in (41) \\
\hline
\end{tabular}

The analytical development in this paper considers all modes of buckling and Fourier series coefficients. The snapping force expressions ((24), (25)) governing the snapthrough behavior contain infinite sums (26), where some of them are independent from the initial shape. The corresponding analytical expressions for these sums were calculated in [55] and are shown in (27). The other infinite sums need to be evaluated depending on the initial shape.

Two case studies are investigated in this section: curved and inclined initial beam shapes. Explicit analytical expressions corresponding to the infinite sums, depending on the initial shape, in both case studies are calculated considering all Fourier series coefficients. However, for other initial shapes, where the calculation of the infinite sums is complex, finite number of Fourier series coefficients can be considered and convergence needs to be checked.

\subsection{Pre-shaped curved beam}

The curved shape (Figure 1.d) is similar to that of the first buckling mode; hence:

$$
\bar{W}=\frac{1}{2} W_{1}
$$

The Fourier series coefficients $C_{0}$ and $C_{j}$ for an initially curved beam are calculated from (14) as follows:

$$
\left\{\begin{array}{l}
C_{0}=\frac{1}{2} \\
C_{j}=-\frac{1}{2} \quad j=1 \\
C_{j}=0 \quad j=\text { else }
\end{array}\right.
$$

The snap-through solution for a pre-shaped curved beam has the form in (18) where $K_{j}$ are calculated by introducing $C_{j}$ from (43) into (23):

$$
\begin{aligned}
& \begin{array}{c}
\text { First kind } \\
N<N_{3}
\end{array} \quad \begin{cases}K_{j}=\frac{4 F-\frac{1}{2} N_{j}^{2} N^{2}}{N_{j}^{2}\left(N^{2}-N_{j}^{2}\right)} j=1 \\
K_{j}=\frac{4 F}{N_{j}^{2}\left(N^{2}-N_{j}^{2}\right)} j=5,9,13 \ldots \\
K_{j}=0 & j=3,7,11 \ldots \\
K_{j}=0 & j=2,4,6 \ldots\end{cases} \\
& \begin{array}{c}
\text { Second kind } \\
N=N_{3}
\end{array} \begin{cases}K_{j}=\frac{4 F-\frac{1}{2} N_{j}^{2} N^{2}}{N_{j}^{2}\left(N^{2}-N_{j}^{2}\right)} & j=1 \\
K_{j}=\frac{4 F}{N_{j}^{2}\left(N^{2}-N_{j}^{2}\right)} & j=5,9,13 \ldots \\
K_{j}=0 & j=7,11,15 \ldots \\
K_{j} \neq 0, C_{j}=0 & j=3 \\
K_{j}=0 & j=2,4,6 \ldots\end{cases}
\end{aligned}
$$

The snapping force expressions for a pre-shaped curved beam for the first kind of solution are given in (24) and (25), where $\Lambda_{b}, \Lambda_{c}$, and $\Lambda_{d}$ are calculated as follows:

$$
\begin{aligned}
& \Lambda_{b}(N)=\frac{-4 \pi^{2}}{\left(N^{2}-4 \pi^{2}\right)^{2}} \\
& \Lambda_{c}(N)=-\frac{\pi^{2} N^{2}}{4} \frac{N^{2}-8 \pi^{2}}{\left(N^{2}-4 \pi^{2}\right)^{2}} \\
& \Lambda_{d}(N)=\frac{N^{2}}{N^{2}-4 \pi^{2}}
\end{aligned}
$$

Also, the snapping force expression in the second kind of solution is obtained from (34) as below:

$$
F_{3}=64 \pi^{2}\left(\frac{4}{3}-\Delta\right)
$$

The third mode constant $K_{3}$ in the second kind of solution is obtained from (37):

$$
K_{3}= \pm \frac{1}{2 \pi} \sqrt{-3 \Delta^{2}+\frac{56}{9} \Delta+\frac{2 \pi^{2}}{9}-\frac{80}{27}-\frac{4 \pi^{2}}{3 Q^{2}}}
$$


The minimum values of $Q$ that allow $N$ to reach $N_{1}, N_{2}$ and $N_{3}$ are obtained from (28):

$$
\begin{aligned}
& \exists N \geq N_{1} \Longleftrightarrow Q \geq \frac{8}{\sqrt{117-7 \pi^{2}}} \approx 1.156 \\
& \exists N \geq N_{2} \Longleftrightarrow Q \gtrsim 1.654 \\
& \exists N=N_{3} \Longleftrightarrow Q \geq \sqrt{\frac{162 \pi^{2}}{27 \pi^{2}+32}} \approx 2.314
\end{aligned}
$$

The condition on $Q$ for the bistability of a pre-shaped curved beam is obtained from (40):

$$
Q \geq \frac{4}{\sqrt{3}} \approx 2.309
$$

The different snapping points coordinates are obtained from the modeling and are expressed as follows:

$$
\begin{aligned}
& F_{\text {top }}, F_{\text {bot }}=64 \pi^{2}\left(\frac{8}{27} \pm \frac{2 \pi}{3} \sqrt{\frac{1}{6}+\frac{16}{81 \pi^{2}}-\frac{1}{Q^{2}}}\right) \\
& \Delta_{\text {top }}, \Delta_{\text {bot }}=\frac{28}{27} \mp \frac{2 \pi}{3} \sqrt{\frac{1}{6}+\frac{16}{81 \pi^{2}}-\frac{1}{Q^{2}}} \\
& \Delta_{m}=\frac{4}{3} \quad \Delta_{\text {end }}=\frac{3}{2}+\sqrt{\frac{1}{4}-\frac{4}{3 Q^{2}}} \\
& \Delta_{\text {final }}=\frac{20}{\pi^{2}} \quad F_{\text {final }}=\frac{3840}{\pi^{2}} \\
& \Delta_{N 1}=\frac{9}{4}-\frac{\pi^{2}}{8} \quad F_{N 1}=2 \pi^{4}
\end{aligned}
$$

The axial force and snapping force curves for a preshaped curved beam (Figures 3, 4, and 5) have been presented in the previous section.

Figure 6 shows a comparison of the snapping force curves for a pair of pre-shaped curved beams in parallel configuration obtained analytically and with a nonlinear FEM model using the ANSYS software. The element "PLANE82" was used in the FEM simulation with static analysis and considering large deformation nonlinearity.

The mesh has been verified to be fine enough for convergence. The beam dimensions considered are $b=25 \mu \mathrm{m}$, $t=10 \mu \mathrm{m}, h=50 \mu \mathrm{m}$, and $l=2 \mathrm{~mm}$, while the material Young's modulus is $E=169 \mathrm{GPa}$. The comparison shows excellent agreement between the two models.

It can be noticed that the solution and expressions derived in this section are similar to those obtained in [55]. This is due to the fact that the solution in $[44,55]$ is approximated as an infinite sum of the modes of buckling of a straight beam (same as for the homogeneous problem (11)) and the initial shape is similar to one of these modes of buckling.

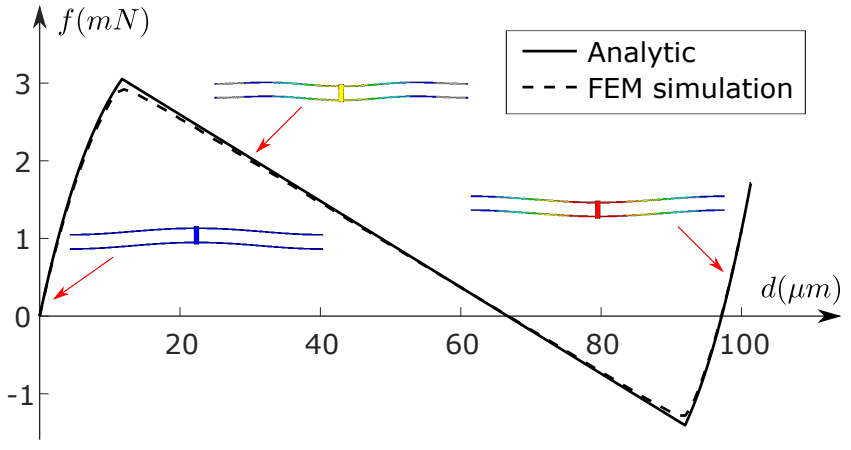

Fig. 6. Snapping force comparison between the analytical and FEM models for a pair of pre-shaped curved beams in parallel configuration.

\subsection{Pre-shaped inclined beam}

The snapping behavior between the curved beam and the inclined beam is very similar qualitatively. However, the conditions, ranges, and levels are changed due to the different initial shape. The initial shape of a pre-shaped inclined beam (Figure 1.c) is described as follows:

$$
\bar{W}(X)=\left\{\begin{array}{cc}
2 X & X \in\left[0, \frac{1}{2}\right] \\
2-2 X & X \in\left[\frac{1}{2}, 1\right]
\end{array}\right.
$$

The Fourier series coefficients $C_{0}$ and $C_{j}$ for an initially inclined beam are calculated from (14) as follows:

$$
\left\{\begin{array}{l}
C_{0}=\frac{1}{2} \\
C_{j}=-\frac{16}{N_{j}^{2}} \quad j=1,5,9 \ldots \\
C_{j}=0 \quad j=\text { else }
\end{array}\right.
$$

The snap-through solution for a pre-shaped inclined beam has the form in (18) where $K_{j}$ are calculated by introducing $C_{j}$ from (52) into (23):

$$
\begin{aligned}
& \begin{aligned}
\text { First kind } \\
N<N_{3}
\end{aligned} \quad \begin{cases}K_{j}=\frac{4\left(F-4 N^{2}\right)}{N_{j}^{2}\left(N^{2}-N_{j}^{2}\right)} j=1,5,9 \ldots \\
K_{j}=0 & j=3,7,11 \ldots \\
K_{j}=0 & j=2,4,6 \ldots\end{cases} \\
& \begin{array}{cl}
\text { Second kind } \\
N=N_{3}
\end{array} \begin{cases}K_{j}=\frac{4\left(F-4 N^{2}\right)}{N_{j}^{2}\left(N^{2}-N_{j}^{2}\right)} & j=1,5,9 \ldots \\
K_{j}=0 & j=7,11,15 \ldots \\
K_{j} \neq 0, C_{j}=0 & j=3 \\
K_{j}=0 & j=2,4,6 \ldots\end{cases}
\end{aligned}
$$

The snapping force equations for a pre-shaped curved beam for the first kind of solution are given in (24) and (25) where $\Lambda_{b}, \Lambda_{c}$ and $\Lambda_{d}$ are infinite sums. However, considering $C_{j}$ in (52), these infinite sums can be expressed in terms of $\Lambda_{a}$ 
and $\Lambda_{e}$, which have their explicit expressions in (27), thus:

$$
\begin{aligned}
& \Lambda_{b}(N)=-8 N^{2} \Lambda_{a}(N)+4 \Lambda_{e}(N) \\
& \Lambda_{c}(N)=16 N^{4} \Lambda_{a}(N)-16 N^{2} \Lambda_{e}(N) \\
& \Lambda_{d}(N)=4 N^{2} \Lambda_{e}(N)
\end{aligned}
$$

Subsequently, the snapping force relation in the second kind of solution is obtained from (34):

$$
F_{3}=64 \pi^{2}(1-\Delta)
$$

The third mode constant $K_{3}$ in the second kind of solution is obtained from (37):

$$
K_{3}= \pm \frac{1}{2 \pi} \sqrt{-3 \Delta^{2}+4 \Delta-\frac{4 \pi^{2}}{3 Q^{2}}}
$$

The minimum values of $Q$, which allow $N$ to reach $N_{1}$, $N_{2}$ and $N_{3}$, can be obtained from (28):

$$
\begin{aligned}
& \exists N \geq N_{1} \Longleftrightarrow Q \geq \frac{\pi^{2}}{4 \sqrt{3}} \approx 1.425 \\
& \exists N \geq N_{2} \Longleftrightarrow Q \gtrsim 2.080 \\
& \exists N=N_{3} \Longleftrightarrow Q \geq \pi \approx 3.142
\end{aligned}
$$

The condition on $Q$ for the bistability of a pre-shaped inclined beam is obtained from (40):

$$
Q \gtrsim 3.196
$$

The snapping force curves for a shallow pre-shaped inclined beam $\left(Q<Q_{3}\right)$ are very similar to those presented in Figure (4). However, the snapping force curve for $\left(Q>Q_{3}\right)$ shows some differences, Figure 7.

The different snapping points coordinates are expressed as follows:

$$
\begin{aligned}
& F_{\text {top }}, F_{\text {bot }}=64 \pi^{2}\left(\frac{1}{3} \pm \frac{2}{3} \sqrt{1-\frac{\pi^{2}}{Q^{2}}}\right) \\
& \Delta_{\text {top }}, \Delta_{\text {bot }}=\frac{2}{3} \mp \frac{2}{3} \sqrt{1-\frac{\pi^{2}}{Q^{2}}} \\
& \Delta_{m}=1 \quad \Delta_{\text {end }}: \frac{N^{2}}{12 Q^{2}}=1-\frac{\tan \frac{N}{4}}{\frac{N}{4}}-\tan ^{2} \frac{N}{4} \\
& \Delta_{\text {final }}=\frac{5}{3} \quad F_{\text {final }}=320 \\
& \Delta_{N 1}=\frac{8}{\pi^{2}} \quad F_{N 1}=16 \pi^{2}
\end{aligned}
$$

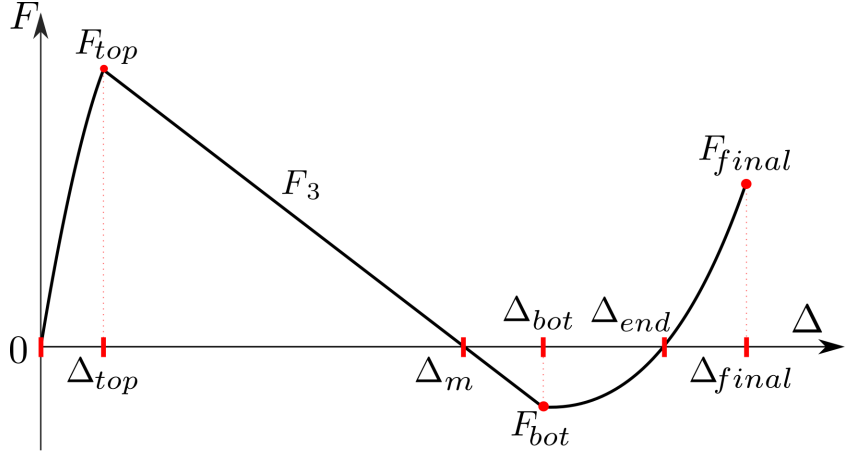

Fig. 7. Snapping force curve for a shallow pre-shaped inclined beam.

The comparison between the analytical and FEM modeling results, for the same beam dimensions and material as in Figure 6, is shown in Figure 8 indicating excellent agreement.

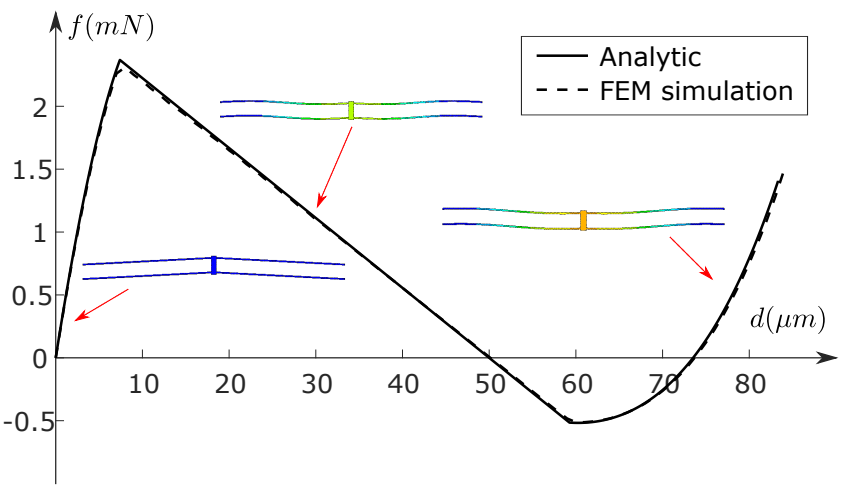

Fig. 8. Snapping force comparison between the analytical and FEM models for a pair of pre-shaped inclined beams in parallel configuration.

In order to further demonstrate the accordance between the analytical modeling and FEM simulations, we choose to verify the accuracy of the bistability condition in (58). Figure 9 shows the snapping force curves obtained from FEM simulations for a pair of inclined beams in parallel configuration with different values of $h$ and the same other dimensions and material as in Figure 8. The values of $h$ are chosen as $Q=3$, 3.2 and 3.5 .

As explained in Section 3.3, the beam with negative snapping force pushes itself to the other side of buckling which leads to bistability. One can notice from Figure 9 that the snapping force curve starts to reach the negative force zone for $Q$ close to 3.2 and higher, in agreement with (58).

\section{Comparison and discussion}

The snap-through behavior and the snapping force curves show some similarities and differences between preshaped curved and inclined beams in terms of forces, positions and $Q$ limits. These difference and similarities are 


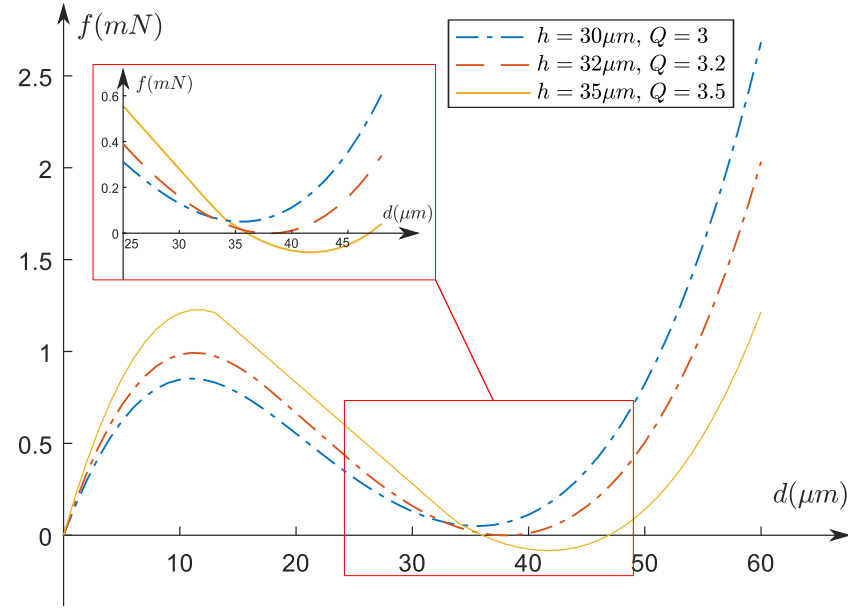

Fig. 9. Snapping force curves for a pair of pre-shaped inclined beams in parallel configuration with different values of $Q$.

investigated and discussed in this section.

\subsection{Conditions on $Q$}

Table 2 shows the $Q$ ratios, for both curved and inclined beams, after which $N$ can reach $N_{1}, N_{2}$ and $N_{3}$ and the minimum $Q$ ratios for bistability in both single and parallel configuration. One can note that the axial force $N$ reaches higher levels and the bistability occurs at lower values of $Q$ for the pre-shaped curved beam. This is more desirable for the design of bistable mechanisms.

Table 2. Minimum $Q$ ratios for reaching $N_{1}, N_{2}$ and $N_{3}$ and for bistability.

\begin{tabular}{|l|c|c|}
\cline { 2 - 3 } \multicolumn{1}{c|}{} & Curved beam & Inclined beam \\
\hline$N_{1}$ & $\frac{8}{\sqrt{117-7 \pi^{2}}} \approx 1.156$ & $\frac{\pi^{2}}{4 \sqrt{3}} \approx 1.425$ \\
\hline$N_{2}$ & $\approx 1.654$ & $\approx 2.080$ \\
\hline$N_{3}$ & $\sqrt{\frac{162 \pi^{2}}{27 \pi^{2}+32}} \approx 2.314$ & $\pi \approx 3.142$ \\
\hline Bistability single & $\approx 5.646$ & Never \\
\hline Bistability parallel & $\frac{4}{\sqrt{3}} \approx 2.309$ & $\approx 3.196$ \\
\hline
\end{tabular}

\subsection{Shallow beams}

For shallow beams with low values of $Q$, a second stable position does not exist but an interesting behavior is noticed. Increasing the value of $Q$, the snapping force curves show a transition between only positive stiffness behavior during deflection to the existence of zero stiffness and negative stiffness zones (Figure 4). This transition in the behavior occurs at the limit of $Q=Q_{1}$ where $N$ starts to reach $N_{1}$. Figure 10 shows a comparison of the snapping force curves during deflection for $Q=Q_{1}$ for both pre-shaped curved and inclined beams.

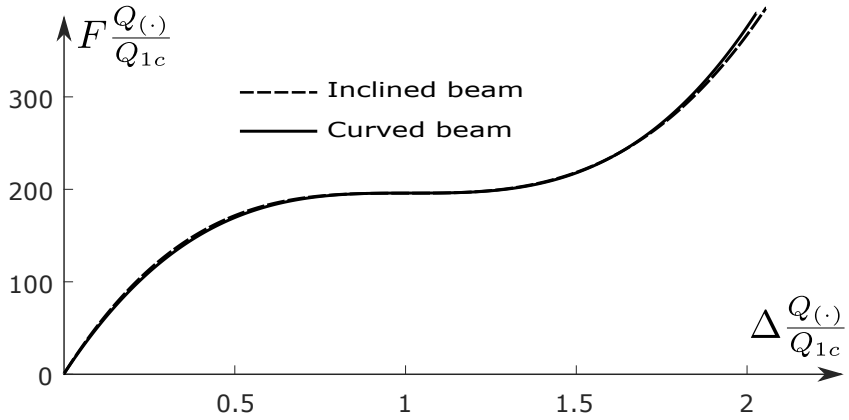

Fig. 10. Comparison of snapping force curves for pre-shaped curved and inclined beams for $Q=Q_{1}$. $Q_{1 c}$ and $Q_{1 i}$ correspond to $Q_{1}$ values for the curved and inclined beams, respectively. $Q_{(\text {.) }}$ refers to $Q_{1 c}$ or $Q_{1 i}$ depending on the corresponding curve.

As $Q_{1}$ is different between the curved and inclined beams, the curve of the inclined beam in Figure 10 is multiplied by a correction factor in force and displacement to enable a suitable frame for comparison. Considering the correction factor and the normalized parameters, the comparison in Figure 10 is between two beam types, which have the same material properties and dimensions, except for $h$ which is different due to the difference in $Q_{1}$. Figure 10 shows an interesting accordance in the snapping force curves between both cases.

\subsection{Bistable beams}

The third mode of buckling get involved during deflection when the critical buckling load is reached and the lateral stiffness becomes negative and constant. Practically, both beam initial shapes under comparison show bistability with the involvement of the third mode of buckling.

Figure 11 shows a comparison in the snapping force curves of the curved and inclined beams when the third mode of buckling is reachable. Considering normalized parameters in the comparison means that both beams have the same material and dimensions. As seen, the inclined beam has lower range of force and displacement.

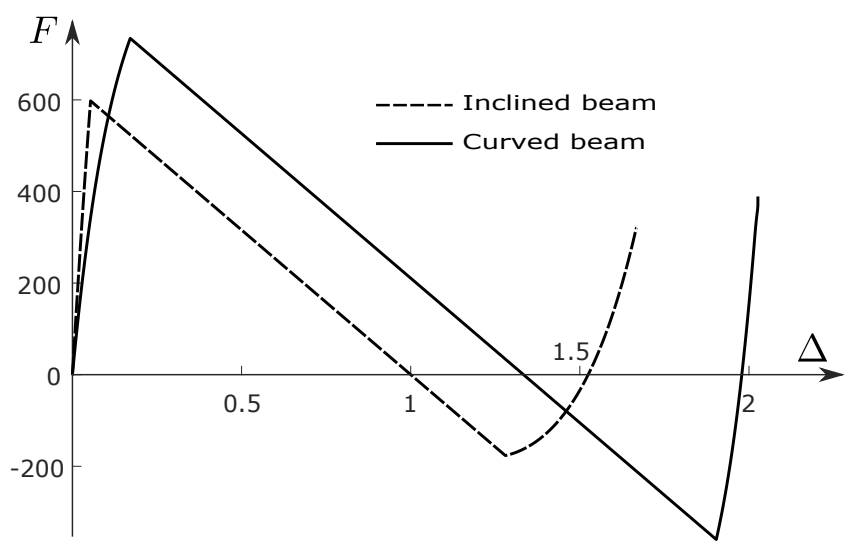

Fig. 11. Comparison of snapping force curves for bistable preshaped beams. 
At the start of deflection, the stiffness of the inclined beam is higher. However, the third mode gets involved earlier, which limits the force level that can be reached.

Further, both beams show exactly the same constant and negative stiffness during the involvement of the third mode of buckling. This is the general case for the stiffness of preshaped beams $S_{3}$ in the second kind of solution (35). Note that the stiffness $S_{3}$ is independent of the initial height $h$.

The normalized force and range of displacement for both pre-shaped beams is highly dependent on $Q$. Figures 12,13 and 14 show the variation with respect to $Q$ of $F_{\text {top }}$, as a measure of the maximum snapping force level, $\Delta_{\text {end }}$, as the distance between the two stable positions, and $F_{b o t}$ over $F_{\text {top }}$ as a measure of the symmetry between the two stable sides. The snap-through behavior is more symmetric when $\left|F_{\text {bot }} / F_{\text {top }}\right|$ is closer to 1 .

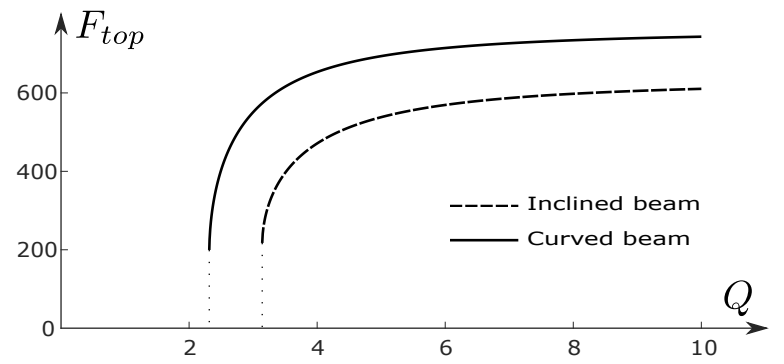

Fig. 12. Variation of $F_{\text {top }}$ with $Q$ for curved and inclined beams.

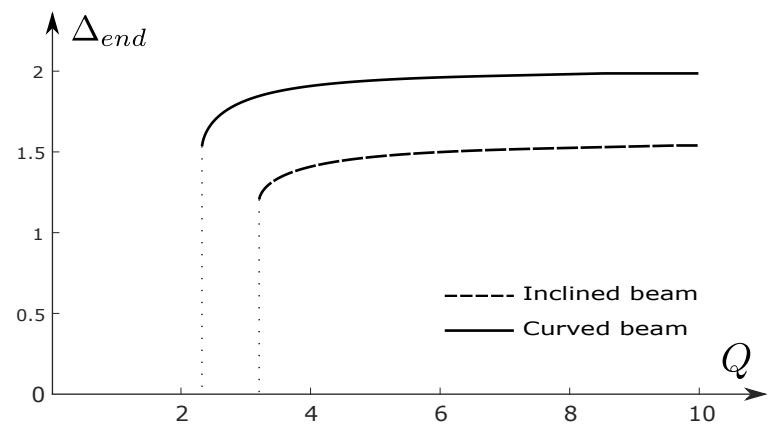

Fig. 13. Variation of $\Delta_{\text {end }}$ with $Q$ for curved and inclined beams.

\section{Conclusions}

In this paper, we derived the snap-through solution and the analytical expressions governing the snapping force of an arbitrarily pre-shaped elastic beam deflected under a midlength lateral point force. The exact solution was calculated based on the classical beam theory and considering all modes of buckling. The snap-through behavior was analyzed based on the analytical expressions. The different snapping points and conditions for negative stiffness, bifurcation of solutions and bistability were clarified. The elements presented in this study constitute design tools for pre-shaped beams.

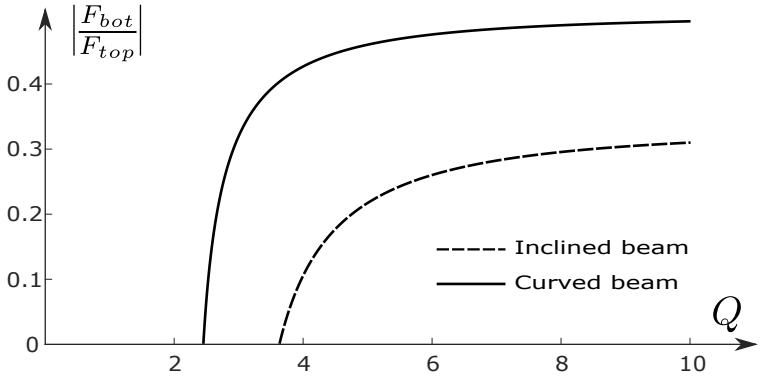

Fig. 14. Variation of $\left|\frac{F_{b o t}}{F_{t o p}}\right|$ with $Q$ for curved and inclined beams.

The calculation procedure for a specific initial shape was demonstrated. The derivations for two initial shapes, curved and inclined, were then investigated. The analytical expressions in both cases were elaborated in explicit formulations considering all modes of buckling. The analytical modeling showed very good agreement with the FEM simulations. Several similarities and differences were noticed in their snap-through behavior. The curved beam shows a wider margin of stability in the second stable side with more symmetric snap-through behavior, while the inclined beam requires larger height-to-thickness ratio to have bistability.

\section{Acknowledgment}

This research has been supported by KAUST research fund.

\section{A Equilibrium equation}

The static equilibrium equation (5) for a pre-shaped beam with a mid-length lateral point force is developed hereinafter based on the classic theory of elastic beams.

Figure 15.a shows a pre-shaped beam with an arbitrarily initial shape before and after deflection due to a mid-length lateral force. Even if the initial shape $(\bar{w}(x))$ is symmetric with respect to the middle of the beam length, the beam shape $(\mathrm{w}(\mathrm{x}))$ is not necessarily symmetric after deflection.

Figure 15.b shows the forces and moments applied on the beam. The force equilibrium in both directions and the moment equilibrium at $\mathrm{A}$ and $\mathrm{B}$ give the following:

$$
\begin{aligned}
P_{A} & =P_{B}=P \\
F_{A} & =F_{B}=\frac{f}{2}
\end{aligned}
$$

Considering small deformation hypothesis, the bending moment $M_{x}$ at the cross section of the beam is expressed as follows:

$$
M_{x}=E I\left(\frac{d^{2} w}{d x^{2}}-\frac{d^{2} \bar{w}}{d x^{2}}\right)
$$

From the static equilibrium, the expression of $M_{x}$ is different before and after beam mid-span where $f$ is applied. 


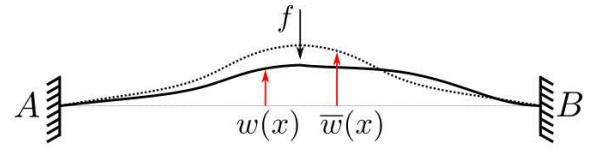

(a)
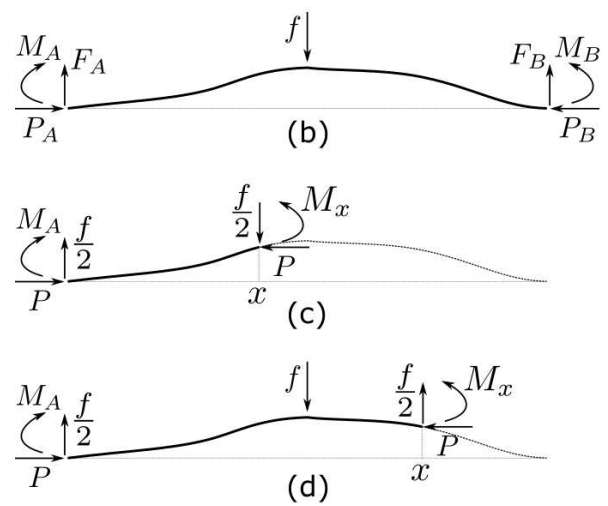

Fig. 15. A pre-shaped beam in deflection (a), forces and moments on the boundaries (b), on a section in the zone $x \in\left[0, \frac{l}{2}\right]$ (c) and on a section in the zone $x \in\left[\frac{l}{2}, l\right]$ (d).

Considering a section in the zone $x \in\left[0, \frac{l}{2}\right]$ (Figure 15.c), the static equilibrium gives the following expression of $M_{x}$ :

$$
M_{x}=M_{A}-P w+\frac{f}{2} x
$$

For a section in the zone $x \in\left[\frac{l}{2}, l\right]$ (Figure 15.d), $M_{x}$ is expressed as follows:

$$
M_{x}=M_{A}-P w+\frac{f}{2}(l-x)
$$

Combining (61), (62) and (63), the equilibrium equation becomes as follows:

$$
E I\left(\frac{d^{2} w}{d x^{2}}-\frac{d^{2} \bar{w}}{d x^{2}}\right)+p w=M_{A}+\frac{f}{2}\left\{\begin{array}{lr}
x & x \in\left[0, \frac{l}{2}\right] \\
l-x & x \in\left[\frac{l}{2}, l\right]
\end{array}\right.
$$

Considering a uniform cross-section and material properties, deriving one time with respect to $x$ to get rid of $M_{A}$, and considering the normalized parameters from (4), the equilibrium equation (64) becomes:

$$
\frac{d^{3} W}{d X^{3}}-\frac{d^{3} \bar{W}}{d X^{3}}+N^{2} \frac{d W}{d X}=\frac{F}{2} \Psi(X)
$$

where $\psi(x)$ is a discontinuous step function:

$$
\Psi(X)=\left\{\begin{array}{cc}
1 & X \in\left[0, \frac{1}{2}\right] \\
-1 & X \in\left[\frac{1}{2}, 1\right]
\end{array}\right.
$$

As the problem has four boundary conditions, the equilibrium equation is represented in the fourth order form:

$$
\frac{d^{4} W}{d X^{4}}-\frac{d^{4} \bar{W}}{d X^{4}}+N^{2} \frac{d^{2} W}{d X^{2}}=\frac{F}{2} \frac{d \Psi}{d X}(X)
$$

The discontinuous step function $\psi(X)$ can be expanded in Fourier series over the beam length as follows:

$$
\Psi(X)=\sum_{j=1,5,9 \ldots} \frac{8}{N_{j}} \sin N_{j} X X \in[0,1]
$$

where $N_{j}=(j+1) \pi$ is the critical buckling load for symmetric modes of buckling as shown in (7).

The Fourier series expansion helps to determine the derivatives of $\psi(X)$ and to quantify the effect of the lateral force $f$ on each mode of buckling. From the derivative of (68), we can notice that:

$$
\frac{d \Psi}{d X}(X)=\delta(X)+\delta(X-1)-2 \delta\left(X-\frac{1}{2}\right)
$$

where $\delta(X)$ is an impulse function over $[0,1]$ domain $\delta(X)=$ $\left\{\begin{array}{ll}1 & X=0 \\ 0 & X \in(0,1]\end{array}\right.$.

Introducing the derivative of (68) into (67), equation (5) is obtained.

$$
\frac{d^{4} W}{d X^{4}}-\frac{d^{4} \bar{W}}{d X^{4}}+N^{2} \frac{d^{2} W}{d X^{2}}=4 F \sum_{j=1,5,9 \ldots} \cos N_{j} X
$$

\section{References}

[1] Zirbel, S. A., Tolman, K. A., Trease, B. P., and Howell, L. L., 2016. "Bistable Mechanisms for Space Applications". PLoS One, 11(12), dec, p. e0168218.

[2] Ben Salem, M., Aiche, G., Rubbert, L., Renaud, P., and Haddab, Y., 2018. "Design of a Microbiota Sampling Capsule using 3D-Printed Bistable Mechanism". In Proc. Annu. Int. Conf. IEEE Eng. Med. Biol. Soc. EMBS, Vol. 2018-July, pp. 4868-4871.

[3] Ando, B., Baglio, S., L'Episcopo, G., and Trigona, C., 2012. "Investigation on Mechanically Bistable MEMS Devices for Energy Harvesting From Vibrations". $J$. Microelectromechanical Syst., 21(4), aug, pp. 779-790.

[4] Vysotskyi, B., Aubry, D., Gaucher, P., Le Roux, X., Parrain, F., and Lefeuvre, E., 2018. "Nonlinear electrostatic energy harvester using compensational springs in gravity field". J. Micromechanics Microengineering, 28(7), jul, p. 074004.

[5] Hajjaj, A. Z., Jaber, N., Ilyas, S., Alfosail, F. K., and Younis, M. I., 2020. "Linear and nonlinear dynamics of micro and nano-resonators: Review of recent advances". Int. J. Non. Linear. Mech., 119, mar, p. 103328. 
[6] Wang, N., Cui, C., Chen, B., Guo, H., and Zhang, X., 2019. "Design of Translational and Rotational Bistable Actuators Based on Dielectric Elastomer". J. Mech. Robot., 11(4), aug.

[7] Hansen, B. J., Carron, C. J., Jensen, B. D., Hawkins, A. R., and Schultz, S. M., 2007. "Plastic latching accelerometer based on bistable compliant mechanisms". Smart Mater. Struct., 16(5), oct, pp. 1967-1972.

[8] Frangi, A., De Masi, B., Confalonieri, F., and Zerbini, S., 2015. "Threshold Shock Sensor Based on a Bistable Mechanism: Design, Modeling, and Measurements". J. Microelectromechanical Syst., 24(6), dec, pp. 20192026.

[9] Jaber, N., Ilyas, S., Shekhah, O., Eddaoudi, M., and Younis, M. I., 2018. "Resonant Gas Sensor and Switch Operating in Air with Metal-Organic Frameworks Coating". J. Microelectromechanical Syst., 27(2), apr, pp. 156-163.

[10] Hajjaj, A., Chappanda, K., Batra, N., Hafiz, M., Costa, P., and Younis, M., 2019. "Miniature pressure sensor based on suspended MWCNT". Sensors Actuators A Phys., 292, jun, pp. 11-16.

[11] Kessler, Y., Ilic, B. R., Krylov, S., and Liberzon, A., 2018. "Flow Sensor Based on the Snap-Through Detection of a Curved Micromechanical Beam". J. Microelectromechanical Syst., 27(6), dec, pp. 945-947.

[12] Truong, Q.-D., Tran, N.-D.-K., and Wang, D.-A., 2017. "Design and characterization of a mouse trap based on a bistable mechanism". Sensors Actuators A Phys., 267, nov, pp. 360-375.

[13] Du, H., Chau, F. S., and Zhou, G., 2018. "Harmonically-Driven Snapping of a Micromachined Bistable Mechanism with Ultra-Small Actuation Stroke”. J. Microelectromechanical Syst., 27(1), feb, pp. 34-39.

[14] Qiu, J., Lang, J. H., Slocum, A. H., and Weber, A. C., 2005. "A bulk-micromachined bistable relay with ushaped thermal actuators". Microelectromechanical Systems, Journal of, 14(5), pp. 1099-1109.

[15] Gomm, T., Howell, L. L., and Selfridge, R. H., 2002. "In-plane linear displacement bistable microrelay". J. Micromechanics Microengineering, 12(3), may, pp. 257-264.

[16] Charlot, B., Sun, W., Yamashita, K., Fujita, H., and Toshiyoshi, H., 2008. "Bistable nanowire for micromechanical memory". Journal of Micromechanics and Microengineering, 18(4), feb, p. 045005.

[17] Hafiz, M. A. A., Kosuru, L., and Younis, M. I., 2016. "Microelectromechanical reprogrammable logic device". Nature communications, 7, p. 11137.

[18] Alfattani, R., and Lusk, C., 2018. "A LaminaEmergent Frustum Using a Bistable Collapsible Compliant Mechanism”. J Mech Design, 140(12), sep, p. 125001.

[19] Chen, G., and Zhang, S., 2011. "Fully-compliant statically-balanced mechanisms without prestressing assembly: concepts and case studies". Mech. Sci., 2(2), aug, pp. 169-174.
[20] Rafsanjani, A., Bertoldi, K., and Studart, A. R., 2019. "Programming soft robots with flexible mechanical metamaterials". Science Robotics, 4(29), p. eaav7874.

[21] Xu, Q., 2017. "Design of a large-stroke bistable mechanism for the application in constant-force micropositioning stage”. J. Mech. Robot., 9(1), dec, p. 011006.

[22] Wang, P., and Xu, Q., 2018. "Design and modeling of constant-force mechanisms: A survey". Mech. Mach. Theory, 119, pp. 1-21.

[23] Sönmez, U., and Tutum, C. C., 2008. "A Compliant Bistable Mechanism Design Incorporating Elastica Buckling Beam Theory and Pseudo-Rigid-Body Model". J Mech Design, 130(4), apr, p. 042304.

[24] Wilcox, D., and Howell, L., 2005. "Fully compliant tensural bistable micromechanisms (FTBM)". J. Microelectromechanical Syst., 14(6), dec, pp. 1223-1235.

[25] Masters, N., and Howell, L., 2003. "A self-retracting fully compliant bistable micromechanism". J. Microelectromechanical Syst., 12(3), jun, pp. 273-280.

[26] Hwang, I.-H., Shim, Y.-S., and Lee, J.-H., 2003. "Modeling and experimental characterization of the chevrontype bi-stable microactuator". J. Micromechanics Microengineering, 13(6), nov, pp. 948-954.

[27] Chen, G., Han, Q., and Jin, K., 2020. "A Fully Compliant Tristable Mechanism Employing Both Tensural and Compresural Segments". J. Mech. Robot., 12(1), feb.

[28] Hussein, H., Bouhadda, I., Mohand-Ousaid, A., Bourbon, G., Le Moal, P., Haddab, Y., and Lutz, P., 2018. "Design and fabrication of novel discrete actuators for microrobotic tasks". Sensors and Actuators A: Physical, 271, pp. 373-382.

[29] Zanaty, M., Vardi, I., and Henein, S., 2018. "Programmable Multistable Mechanisms: Synthesis and Modeling". J Mech Design, 140(4), feb, p. 042301.

[30] Che, K., Yuan, C., Wu, J., Jerry Qi, H., and Meaud, J., 2016. "Three-Dimensional-Printed Multistable Mechanical Metamaterials With a Deterministic Deformation Sequence". J Appl Mech, 84(1), oct, p. 011004.

[31] Chen, G., Gou, Y., and Zhang, A., 2011. "Synthesis of compliant multistable mechanisms through use of a single bistable mechanism". J Mech Design, 133(8), p. 081007.

[32] Chalvet, V., Haddab, Y., and Lutz, P., 2013. "A microfabricated planar digital microrobot for precise positioning based on bistable modules". IEEE Transactions on Robotics, 29(3), pp. 641-649.

[33] Vangbo, M., 1998. "An analytical analysis of a compressed bistable buckled beam". Sensors Actuators A Phys., 69(3), sep, pp. 212-216.

[34] Alkharabsheh, S. A., and Younis, M. I., 2013. "Statics and dynamics of mems arches under axial forces". Journal of Vibration and Acoustics, 135(2), p. 021007.

[35] Camescasse, B., Fernandes, A., and Pouget, J., 2013. "Bistable buckled beam: Elastica modeling and analysis of static actuation". Int. J. Solids Struct., 50(19), sep, pp. 2881-2893.

[36] Gerson, Y., Krylov, S., and Ilic, B., 2010. "Electrothermal bistability tuning in a large displacement micro ac- 
tuator". Journal of Micromechanics and Microengineering, 20(11), p. 112001.

[37] Michael, A., Kwok, C. Y., Yu, K., and Mackenzie, M. R., 2008. "A novel bistable two-way actuated outof-plane electrothermal microbridge". Journal of $\mathrm{Mi}$ croelectromechanical Systems, 17(1), pp. 58-69.

[38] Chiao, M., and Lin, L., 2000. "Self-buckling of micromachined beams under resistive heating". Journal of Microelectromechanical systems, 9(1), pp. 146-151.

[39] Lorenzoni, M., Llobet, J., and Perez-Murano, F., 2018. "Study of buckling behavior at the nanoscale through capillary adhesion force". Appl. Phys. Lett., 112(19), may, p. 193102.

[40] Pane, I. Z., and Asano, T., 2008. "Investigation on Bistability and Fabrication of Bistable Prestressed Curved Beam". Jpn. J. Appl. Phys., 47(6), jun, pp. 5291-5296.

[41] Fang, W., and Wickert, J. A., 1994. "Post buckling of micromachined beams". J. Micromechanics Microengineering, 4(3), sep, pp. 116-122.

[42] Tajaddodianfar, F., Pishkenari, H. N., Yazdi, M. R. H., and Miandoab, E. M., 2015. "Size-dependent bistability of an electrostatically actuated arch NEMS based on strain gradient theory". J. Phys. D. Appl. Phys., 48(24), jun, p. 245503.

[43] Krylov, S., Ilic, B. R., and Lulinsky, S., 2011. "Bistability of curved microbeams actuated by fringing electrostatic fields". Nonlinear Dyn., 66(3), nov, pp. 403-426.

[44] Qiu, J., Lang, J., and Slocum, A., 2004. "A CurvedBeam Bistable Mechanism". J. Microelectromechanical Syst., 13(2), apr, pp. 137-146.

[45] Hussein, H., Le Moal, P., Younes, R., Bourbon, G., Haddab, Y., and Lutz, P., 2019. "On the design of a preshaped curved beam bistable mechanism". Mech. Mach. Theory, 131, pp. 204-217.

[46] Park, S., and Hah, D., 2008. "Pre-shaped buckled-beam actuators: Theory and experiments". Sensors Actuators A Phys., 148(1), nov, pp. 186-192.

[47] Aimmanee, S., and Tichakorn, K., 2018. "Piezoelectrically induced snap-through buckling in a buckled beam bonded with a segmented actuator". J. Intell. Mater. Syst. Struct., 29(9), may, pp. 1862-1874.

[48] Camescasse, B., Fernandes, A., and Pouget, J., 2014. "Bistable buckled beam and force actuation: Experimental validations”. Int. J. Solids Struct., 51(9), may, pp. 1750-1757.

[49] Cazottes, P., Fernandes, A., Pouget, J., and Hafez, M., 2009. "Bistable buckled beam: modeling of actuating force and experimental validations". J Mech Design, 131(10), p. 101001.

[50] Chen, Q., Zhang, X., Zhang, H., Zhu, B., and Chen, B., 2019. "Topology optimization of bistable mechanisms with maximized differences between switching forces in forward and backward direction". Mech. Mach. Theory, 139, sep, pp. 131-143.

[51] Gao, R., Li, M., Wang, Q., Zhao, J., and Liu, S., 2018. "A novel design method of bistable structures with required snap-through properties". Sensors Actuators A
Phys., 272, apr, pp. 295-300.

[52] Huang, Y., Zhao, J., and Liu, S., 2016. "Design optimization of segment-reinforced bistable mechanisms exhibiting adjustable snapping behavior". Sensors and Actuators A: Physical, 252, pp. 7-15.

[53] Tran, N. D. K., and Wang, D.-A., 2017. "Design of a crab-like bistable mechanism for nearly equal switching forces in forward and backward directions". Mech. Mach. Theory, 115, pp. 114-129.

[54] Chen, Q., Zhang, X., and Zhu, B., 2018. "Design of buckling-induced mechanical metamaterials for energy absorption using topology optimization". Struct. Multidiscip. Optim., 58(4), oct, pp. 1395-1410.

[55] Hussein, H., Le Moal, P., Bourbon, G., Haddab, Y., and Lutz, P., 2015. "Modeling and Stress Analysis of a Pre-Shaped Curved Beam: Influence of High Modes of Buckling". Int. J. Appl. Mech., 07(04), p. 1550055.

[56] Williams, M. D., van Keulen, F., and Sheplak, M., 2012. "Modeling of Initially Curved Beam Structures for Design of Multistable MEMS”. J Appl Mech, 79(1), jan, p. 011006.

[57] Palathingal, S., and Ananthasuresh, G. K., 2019. "Analysis and Design of Fixed-Fixed Bistable Arch-Profiles Using a Bilateral Relationship". J. Mech. Robot., 11(3), jun.

[58] Zhao, J., Jia, J., He, X., and Wang, H., 2008. "Postbuckling and Snap-Through Behavior of Inclined Slender Beams". J Appl Mech, 75(4), jul, p. 041020.

[59] Zhang, A., and Chen, G., 2013. "A Comprehensive Elliptic Integral Solution to the Large Deflection Problems of Thin Beams in Compliant Mechanisms". $J$ Mech Robot, 5(2), mar, p. 021006.

[60] Holst, G. L., Teichert, G. H., and Jensen, B. D., 2011. "Modeling and Experiments of Buckling Modes and Deflection of Fixed-Guided Beams in Compliant Mechanisms". J Mech Design, 133(5), may, p. 051002.

[61] Kim, C., 2011. "Curve Decomposition Analysis for Fixed-Guided Beams With Application to Statically Balanced Compliant Mechanisms". In Vol. 6 35th Mech. Robot. Conf. Parts A B, ASME, pp. 149-158.

[62] Chen, G., and Ma, F., 2014. "Kinetostatic Modeling of Fully Compliant Bistable Mechanisms Using Timoshenko Beam Constraint Model". J Mech Design, 137(2), feb, p. 022301.

[63] Awtar, S., Slocum, A. H., and Sevincer, E., 2007. "Characteristics of Beam-Based Flexure Modules". J Mech Design, 129(6), jun, p. 625.

[64] Ma, F., and Chen, G., 2017. "Bi-BCM: A Closed-Form Solution for Fixed-Guided Beams in Compliant Mechanisms". J. Mech. Robot., 9(1), feb.

[65] Chen, G., Ma, F., Hao, G., and Zhu, W., 2019. "Modeling Large Deflections of Initially Curved Beams in Compliant Mechanisms Using Chained Beam Constraint Model". J. Mech. Robot., 11(1), feb.

[66] Nayfeh, A. H., and Emam, S. A., 2008. "Exact solution and stability of postbuckling configurations of beams". Nonlinear Dyn., 54(4), dec, pp. 395-408. 